\title{
Wolff-Parkinson-White Syndrome Concomitant with Idiopathic Ventricular Fibrillation Associated with Inferior Early Repolarization
}

\author{
Naohiko Takahashi ${ }^{1}$, Tetsuji Shinohara ${ }^{2}$, Masahide Hara $^{2}$ and Tetsunori Saikawa ${ }^{1}$
}

\begin{abstract}
We encountered a 39-year-old man with documented ventricular fibrillation (VF). His ECGs showed intermittent Wolff-Parkinson-White (WPW) syndrome pattern. During electrophysiological study, no ventricular preexcitation was observed. An accessory pathway located at the posterior mitral annulus was identified, and successfully eliminated by radiofrequency catheter ablation. VF was not induced. His ECGs in the absence of delta waves demonstrated early repolarization in the inferior leads. This case raises the possibility that patients with manifest WPW syndrome may have an arrhythmogenic substrate associated with early repolarization, and the characteristic $\mathrm{J}$ waves can be masked by the presence of ventricular preexcitation.
\end{abstract}

Key words: Wolff-Parkinson-White syndrome, J wave, early repolarization, ventricular fibrillation

(Intern Med 51: 1861-1864, 2012)

(DOI: 10.2169/internalmedicine.51.7353)

\section{Introduction}

Patients with Wolff-Parkinson-White (WPW) syndrome can suddenly die $(1,2)$. The underlying mechanism has been accepted to be atrial fibrillation (AF) with a very rapid ventricular rate, deteriorating into ventricular fibrillation (VF) $(1,2)$. In contrast, early repolarization in the inferior or inferolateral leads is associated with so-called idiopathic VF (3). Here, we present a case with documented VF who had been diagnosed as having WPW syndrome and paroxysmal supraventricular tachycardia (SVT). His ECGs showed intermittent WPW syndrome pattern. His ECGs in the absence of delta waves demonstrated early repolarization in the inferior leads. We therefore attributed the documented VF to inferior early repolarization rather than deterioration from AF with rapid ventricular response. The association of WPW syndrome and documented idiopathic VF associated with inferior early repolarization has not been previously reported. This case raises the cautionary point that arrhythmic and/or syncope events observed in patients with WPW syndrome could be associated with masked inferior or infero- lateral early repolarization.

\section{Case Report}

A 39-year-old man experienced sudden syncope during lounging on the sofa after taking a bath at midnight. The responding firemen confirmed the patient's VF (Fig. 1A). The initial three applications of direct-current shocks failed to defibrillate the VF episode. The VF was successfully defibrillated by the fourth shock (Fig. 1B), which was 11 minutes after the arrival of firemen. The monitor ECG immediately after the fourth shock showed AF with heart rate around $120 / \mathrm{min}$, with no signs of ventricular preexcitation (Fig. 1C). The 12-lead ECG recorded at the hospital, which was 16 minutes after the VF defibrillation, demonstrated sinus rhythm with ventricular preexcitation, a typical type A pattern of WPW syndrome (Fig. 2). He regained consciousness two days later. Two years previously, he had first experienced syncope for several minutes. The following year, he was diagnosed as having WPW syndrome by 12-lead ECG (Fig. 3A). Repeatedly-recorded ECGs revealed his delta waves to be intermittent. SVT was also documented

${ }^{1}$ Department of Laboratory Examination and Diagnostics, Faculty of Medicine, Oita University, Japan and ${ }^{2}$ Department of Internal Medicine 1, Faculty of Medicine, Oita University, Japan

Received for publication January 19, 2012; Accepted for publication April 15, 2012

Correspondence to Dr. Naohiko Takahashi, takanao@oita-u.ac.jp 
A

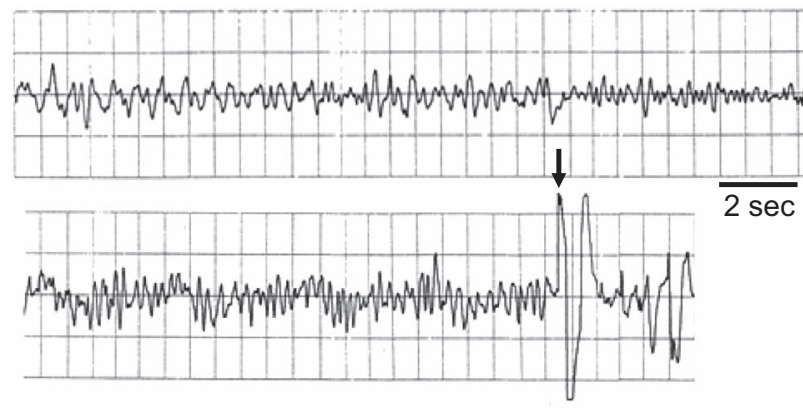

C

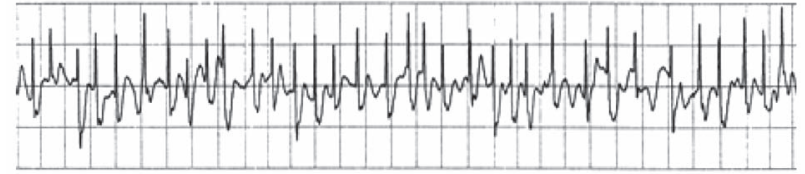

Figure 1. Series of monitor ECG connected to automated external defibrillator. A: Ventricular fibrillation (VF) confirmed by the firemen 10 minutes after the initiation of syncope. B: The application of fourth direct-current shock (arrow) defibrillated the VF. C: Atrial fibrillation immediately after the VF defibrillation. No signs of Wolff-Parkinson-White (WPW) syndrome were observed. B and C are continuous recordings. Note that ECGs were transversely compressed.

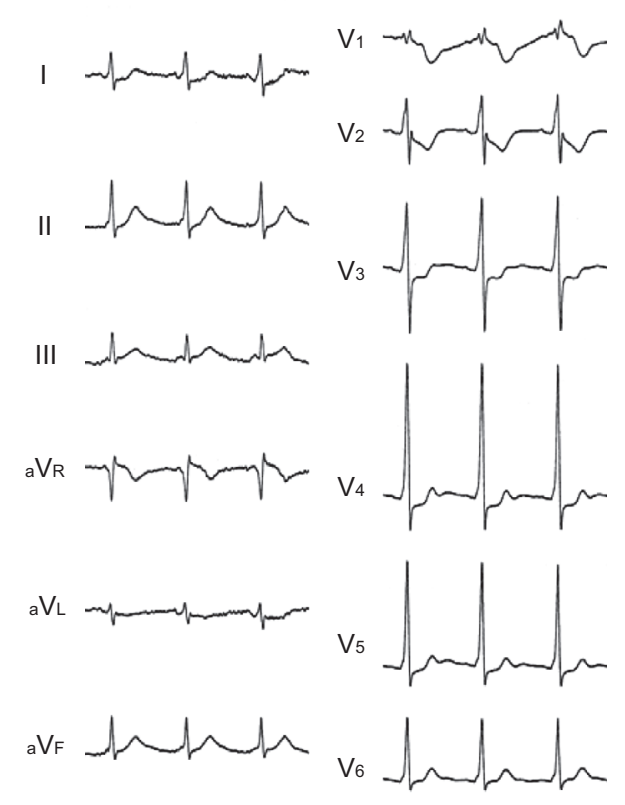

Figure 2. The 12-lead ECG recorded 16 minutes after the VF defibrillation. Sinus rhythm with ventricular preexcitation, a typical type A pattern of WPW syndrome was recorded. $S T$ depression observed in $V_{2}$ to $V_{5}$ may be due to global myocardial ischemia.

\section{(Fig. 3B).}

One month after the VF episode, he was referred to our hospital for a thorough examination. He had no structural heart disease as evaluated by physical examination, chest $\mathrm{X}$ ray, and echocardiography. Electrophysiological study was performed with the patient in the fasting and unsedated state. At the start of the study, no ventricular preexcitation was observed. The ventricular preexcitation did not become manifest by any atrial or ventricular programmed stimulation. Thus, the refractory period of antegrade accessory pathway conduction could not be evaluated. SVT was reproducibly induced, during which retrograde conduction via an accessory pathway located at the posterior mitral annulus was confirmed, leading to a definite diagnosis of atrioventricular reciprocating tachycardia (AVRT). The refractory period of retrograde accessory pathway conduction was 340 msec. During right ventricular apex pacing, radiofrequency ablation was performed, resulting in the elimination of retrograde accessory pathway conduction. Following the successful ablation, VF inducibility was tested. VF was not induced by any programmed ventricular stimulations from the right ventricular apex or from the right ventricular outflow tract using 3 extrastimuli. The effective refractory period of the right ventricle was revealed to be $190 \mathrm{msec}$ or less. His coronary angiogram revealed no organic stenosis, and a vasospasm provocation test using ergonovine infusion into the right coronary artery was negative. A provocation test using intravenous administration of the sodium channel blocker (pilsicainide $50 \mathrm{mg}$ ) caused no apparent ECG changes. After obtaining written informed consent, an implantable cardioverter-defibrillator (ICD) was implanted.

His ECGs after the catheter ablation showed early repolarization/J wave in leads II, III and aVF (Figs. 3C and 3D). The QT/QTc interval was 424/407 msec. His previouslyrecorded ECGs in the absence of delta waves showed essentially the same early repolarization in the inferior leads. During the 4-year follow-up, no recurrence of VF, ECG pattern of WPW syndrome or paroxysmal SVT was observed.

\section{Discussion}

Because of the previous diagnosis of WPW syndrome, the documented VF in this case was initially considered to be due to AF with a very rapid ventricular response. However, ECG pattern of manifest WPW syndrome was intermittent. In addition, antegrade conduction via accessory pathway was not observed by any programmed stimulations during electrophysiological study. These findings suggested little possibility of AF with a very rapid ventricular rate as a cause of the documented VF. His ECGs in the absence of delta waves demonstrated early repolarization in the inferior leads. We therefore attributed the documented VF to inferior early repolarization, although the possibility that VF deteriorated from AF with a very rapid ventricular rate could not be excluded exclusively.

There have been several reports which demonstrate the coexistence of WPW syndrome and Brugada syndrome (4-6). Bodegas et al. (4) firstly described 32-year-old man with occult WPW syndrome concomitant with asymptomatic Brugada syndrome. Ohkubo et al. (5) also reported a 29-year-old male manifest WPW syndrome patient concomitant with asymptomatic Brugada syndrome. Although both of those patients were asymptomatic $(4,5)$, they re- 
A

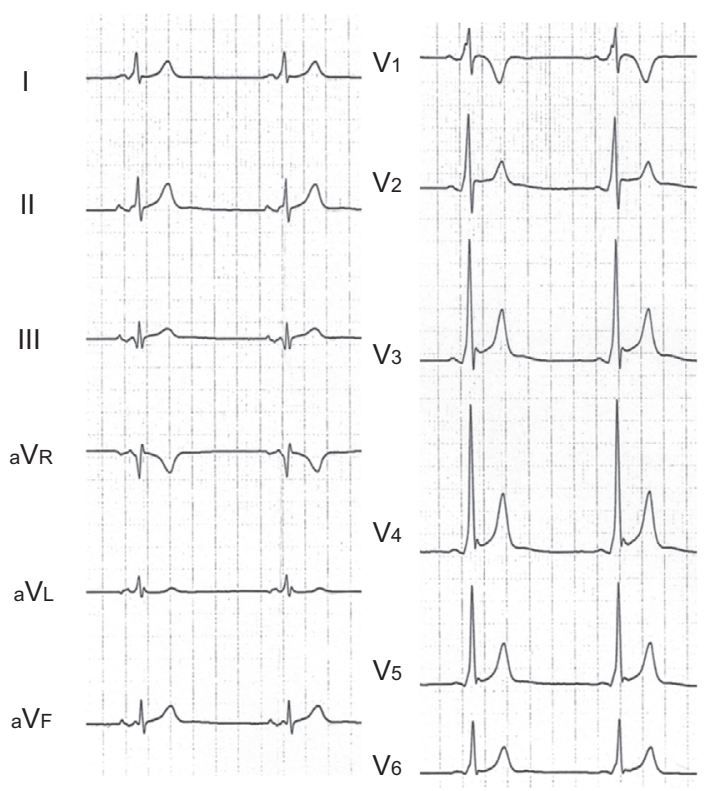

C

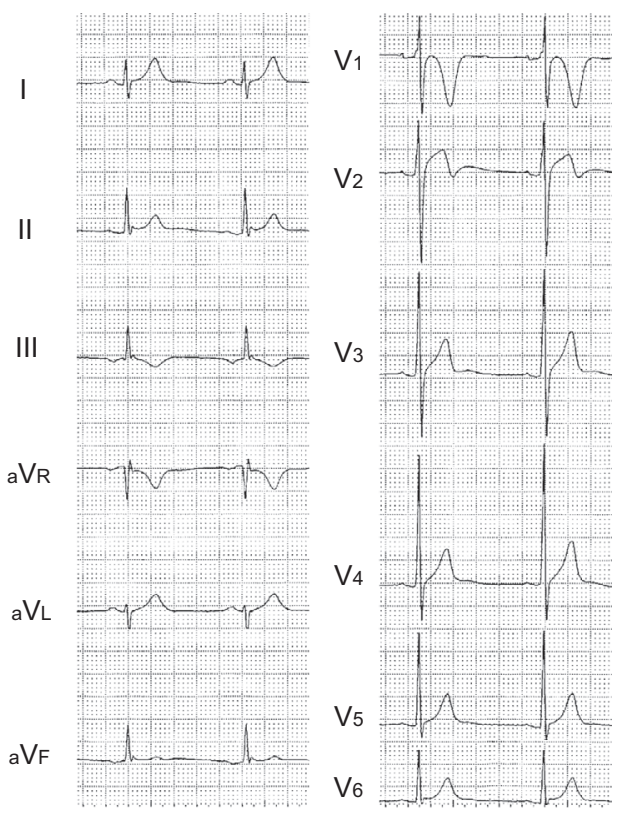

III

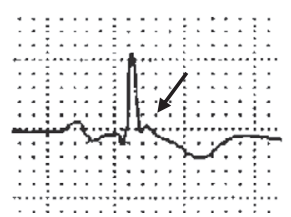

$\mathrm{a} \mathrm{V}_{\mathrm{F}}$

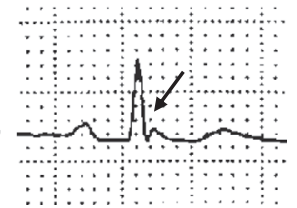

Figure 3. The ECGs before (A, B) and after the catheter ablation (C, D). A: ECG recoded one year before the VF episode showing type A pattern of WPW syndrome. $J$ waves were observed in $V_{2}$ to $V_{5}$. B: Documented SVT. Heart rate was 140/min C: ECG recorded 6 months after the catheter ablation. D: Magnified ECGs in leads II, III and $\mathbf{a V}_{\mathrm{F}}$ in panel $\mathrm{C}$.

ceived ICD implantation because of inducible VF during electrophysiologic study. Kim et al. (6) described a 20-yearold man with occult WPW syndrome for whom theuse of intravenous diltiazem and metoprorol for the rate control of paroxysmal AF with rapid ventricular responses precipitated VF (6). The prevalence of early repolarization in patients with WPW syndrome before and after successful ablation has been reported $(7,8)$. Because early repolarization in the presence of ventricular preexcitation was consistently demonstrated in the leads with positive deflection of the initial part of the delta wave, Mizumaki et al. concluded that early repolarization in patients with WPW syndrome may be related to the early depolarization through accessory pathways (7). Yagihara also attributed early repolarization during manifest preexcitation in patients with WPW syndrome to early depolarization of ventricular myocardium (8). In both studies $(7,8)$, early repolarization was observed after successful accessory pathway ablation in some patients. The mechanisms of early repolarization after ablation could be theoretically explained by the presence of a transient outward current (Ito)-mediated prominent action potential notch in the epicardium, but not endocardium, provides a voltage gradient, which manifests as J waves (9). The present case, in the presence of delta waves, showed $\mathrm{J}$ waves in leads $\mathrm{V}_{2}$ to $\mathrm{V}_{5}$, but not in leads II, III and aVF. In other words, the "true" early repolarization in the inferior leads was masked by the presence of delta waves. This is the first report demonstrating the coexistence of WPW syndrome and documented idiopathic VF associated with inferior early repolarization. The lack of $\mathrm{J}$ waves soon after the resuscitation (Fig. 2) may be explained by myocardial ischemia due to cardiopulmonary arrest, evidenced by the ST depression in extended leads. The present case is of clinical importance as it raises the cautionary point that arrhythmic and/or syncope events observed in patients with WPW syndrome could be associated with masked inferolateral early repolarization. In this regard, Eckardt et al. (10) carried out an electrophysiological test in 35 consecutive patients with Brugada syndrome, and found that ten patients (29\%) have SVT. In two patients, AVRT using accessory pathways was identified (10). One of those two patients had a history of sudden cardiac death (11). Based on these observations, the authors indicated that in patients with SVT and aborted sudden cardiac death or syncope not related to SVT, the Brugada syndrome should be considered a possible additional electrophysiological abnormality $(8,9)$. We again emphasize the possibility that patients with manifest WPW syndrome may have an arrhythmogenic substrate associated with early repo- 
larization, and the characteristic $\mathrm{J}$ waves can be masked by the presence of ventricular preexcitation.

The authors state that they have no Conflict of Interest (COI).

\section{References}

1. Dreifus LS, Haiat R, Watanabe Y, Arriaga J, Reitman N. Ventricular fibrillation. A possible mechanism of sudden death in patients and Wolff-Parkinson-White syndrome. Circulation 43: 520-527, 1971.

2. Dreifus LS, Wellens HJ, Watanabe Y, Kimbiris D, Truex R. Sinus bradycardia and atrial fibrillation associated with the WolffParkinson-White syndrome. Am J Cardiol 38: 149-156, 1976.

3. Haïssaguerre M, Derval N, Sacher F, et al. Sudden cardiac arrest associated with early repolarization. N Engl J Med 358: 20162023, 2008.

4. Bodegas AI, Arana JI, Vitoria Y, Arriandiaga JR, Barrenetxea JI. Brugada syndrome in a patient with accessory pathway. Europace 4: 87-89, 2002.

5. Ohkubo K, Watanabe I, Okumura Y, et al. Wolff-Parkinson-White syndrome concomitant with asymptomatic Brugada syndrome. Pacing Clin Electrophysiol 27: 109-111, 2004.

6. Kim RJ, Gerling BR, Kono AT, Greenberg ML. Precipitation of ventricular fibrillation by intravenous diltiazem and metoprolol in a young patient with occult Wolff-Parkinson-White syndrome. Pacing Clin Electrophysiol 31: 776-779, 2008.

7. Mizumaki K, Nishida K, Iwamoto J, et al. Early repolarization in Wolff-Parkinson-White syndrome: prevalence and clinical significance. Europace 13: 1195-1200, 2011.

8. Yagihara N, Sato A, Iijima K, et al. The prevalence of early repolarization in Wolff-Parkinson-White syndrome with a special reference to $\mathrm{J}$ waves and the effects of catheter ablation. J Electrocardiol 45: 36-42, 2012.

9. Yan GX, Antzelevitch C. Cellular basis for the electrocardiographic J wave. Circulation 93: 372-379, 1996.

10. Eckardt L, Kirchhof P, Loh P. Brugada syndrome and supraventricular tachyarrhythmias: a novel association? J Cardiovasc Electrophysiol 12: 680-685, 2001.

11. Eckardt L, Kirchhof P, Johna R, Haverkamp W, Breithardt G, Borggrefe M. Wolff-Parkinson-White syndrome associated with Brugada syndrome. Pacing Clin Electrophysiol 24 (9 Pt 1): 14231424, 2001.

(C) 2012 The Japanese Society of Internal Medicine http://www.naika.or.jp/imindex.html 